University of Wollongong

Research Online

Faculty of Engineering and Information

Faculty of Engineering and Information

Sciences - Papers: Part A

Sciences

$1-1-2013$

Influence of external optical feedback on the alpha factor of semiconductor lasers

Yanguang Yu

University of Wollongong, yanguang@uow.edu.au

Jiangtao Xi

University of Wollongong, jiangtao@uow.edu.au

Follow this and additional works at: https://ro.uow.edu.au/eispapers

Part of the Engineering Commons, and the Science and Technology Studies Commons

Research Online is the open access institutional repository for the University of Wollongong. For further information contact the UOW Library: research-pubs@uow.edu.au 


\title{
Influence of external optical feedback on the alpha factor of semiconductor lasers
}

\begin{abstract}
This Letter presents the results revealing the influence of external optical feedback (EOF) on the alpha factor, or the linewidth enhancement factor, of semiconductor lasers operating on single mode. First, a method is proposed for the measurement of the alpha over a wide range of optical feedback level, which provides an effective way for investigating the dependence of the alpha on laser operating conditions. Second, experimental investigation based on the proposed method is performed on a GaAlAs laser diode with a multiquantum well structure. It is discovered that the alpha value remains approximately constant with increasing injection current, but has a strong dependence on EOF.
\end{abstract}

\section{Keywords}

semiconductor, factor, lasers, alpha, influence, feedback, optical, external

Disciplines

Engineering | Science and Technology Studies

\section{Publication Details}

Y. Yu \& J. Xi, "Influence of external optical feedback on the alpha factor of semiconductor lasers," Optics Letters, vol. 38, (11) pp. 1781-1783, 2013. 


\title{
Influence of external optical feedback on the alpha factor of semiconductor lasers
}

\author{
Yanguang $\mathrm{Yu}^{*}$ and Jiangtao $\mathrm{Xi}$ \\ School of Electrical, Computer and Telecommunications Engineering, University of Wollongong, \\ Northfields Ave, Wollongong, NSW 2522, Australia \\ *Corresponding author: yanguang@uow.edu.au
}

Received September 4, 2012; revised April 22, 2013; accepted April 23, 2013; posted April 23, 2013 (Doc. ID 175628); published May 20, 2013

\begin{abstract}
This Letter presents the results revealing the influence of external optical feedback (EOF) on the alpha factor, or the linewidth enhancement factor, of semiconductor lasers operating on single mode. First, a method is proposed for the measurement of the alpha over a wide range of optical feedback level, which provides an effective way for investigating the dependence of the alpha on laser operating conditions. Second, experimental investigation based on the proposed method is performed on a GaAlAs laser diode with a multiquantum well structure. It is discovered that the alpha value remains approximately constant with increasing injection current, but has a strong dependence on EOF. () 2013 Optical Society of America

OCIS codes: (120.3180) Interferometry; (280.3420) Laser sensors; (140.5960) Semiconductor lasers.

http://dx.doi.org/10.1364/OL.38.001781
\end{abstract}

The linewidth enhancement factor (also referred to as the alpha factor, the $\alpha$ parameter, Henry factor, the chirp factor, or the phase-amplitude coupling factor) is one of fundamental parameters for semiconductor lasers (SLs). The parameter was introduced by Henry in 1982 [1]. Its value is very important for describing many aspects of laser behavior, such as spectral effects, modulation response, injection locking, and the response to external optical feedback (EOF) [2]. Therefore, it is of significant interest to know the value of this parameter since knowledge of alpha is required both for analysis and design. Various methods have been developed for experimental determination of the alpha parameter, which can be categorized as the linewidth measurement method, current modulations method, optical injection, and optical feedback [2,3]. Some recent work is also reported, such as the method based on temporal profile and the instantaneous frequency (chirp) of the pulses generated by gain switching [4] and the technique using a Mach-Zehnder interferometer [5].

A challenging aspect with the alpha parameter is that its value varies significantly with respect to different SL structures and to different measurement methods. For example, for semiconductor quantum well (QW) lasers, it is commonly observed that the alpha falls within the range from 2 to 6 [2, $\underline{6}]$. For different quantum dot (QD) lasers, the alpha can spread over a range from 0 to 60 $[4,7]$. When applying different measurement techniques onto a single QW laser, the alpha value is observed to spread from 3.5 to 6.2 [3]. The spread in the reported alpha values raises a question: what factors influence this parameter? Some early but important results were reported in [2], showing that, for QW lasers, the alpha varies considerably with photoenergy and carrier density, but it remains approximately constant at the gain peak energy. Also, recent research on QD lasers reveals that the alpha has strong connections to injection current and the properties of QD [6-10].

This paper aims to investigate the influence of EOF on the alpha parameter. We noticed a recent report [11] showing that the alpha value for a solitary QW laser decreases when the laser is subject to certain EOF, indicating that
EOF does influence the alpha. However, to the best of our knowledge, there is not yet a report in literature investigating how the alpha changes with the EOF. In fact, EOF has significant influence on SL behaviors $[\underline{12}, \underline{13}]$, and intensive research on the topic has created many technologies. One of them is called self-mixing interferometry (SMI), which can be used for the measurement of displacement, vibration, velocity, and distance [14]. Based on the SMI, a set of techniques were also developed for the measurement of the alpha for single-mode lasers over the past few years [15-17]. The relative strength of EOF can be described by a parameter called optical feedback parameter denoted by $C$ [13]. SMI-based methods are able to determine both alpha and $C$, hence offering a possible way to study the influence of EOF on the alpha. Unfortunately, all the existing SMI-based methods can only measure the alpha within a certain narrow range of $C$. For example, the method in [15] only works if the optical feedback falls within the range with $1<C<3$. The methods in $[16,17]$ are only applicable when $C<1$. The work in [18] is able to measure $C$ over a large range of EOF but fails to work out the alpha. In the following, a novel algorithm is proposed to lift the above limitations with which experimental investigation can be carried out to study the dependence of the alpha on the SL operation condition, including EOF as well as the injection current.

An SMI consists of an SL, a focus lens, and an external target. When a small fraction of the light is back-reflected or backscattered by the target, it reenters the laser cavity and causes modulation to the power emitted by the SL. The modulated power is detected as an SMI signal which can be used to retrieve the two parameters alpha and $C$. A set of mathematical models [15-19] for describing the SMI signal is derived from Lang and Kobayashi (L-K) equations [12], shown as follows:

$$
\begin{gathered}
\phi_{F}(t)=\phi_{0}(t)-C \cdot \sin \left[\phi_{F}(t)+\arctan (\alpha)\right], \\
g(t)=\cos \left(\phi_{F}(t)\right), \\
p(t)=p_{0}[1+m \cdot g(t)],
\end{gathered}
$$


where $t$ is the time index. Equation (1) gives the relationship between $\phi_{0}(t)$ and $\phi_{F}(t)$, denoting, respectively, the light phase of the unperturbed SL and the phase of the SL with optical feedback. $\phi_{0}(t)=4 \pi l(t) / \lambda_{0}$, where $\lambda_{0}$ is the lasing wavelength of the solitary SL and $l(t)$ represents the distance between the front facet of the laser and the target surface. $g(t)$ captures the influence of EOF on the laser power $p(t) . m$ is the modulation index and $p_{0}$ represents the laser power for a solitary laser. The two parameters in Eq. (1), $\alpha$ and $C$, are the alpha and optical feedback parameter, respectively.

By expanding $\sin \left[\phi_{F}(t)+\arctan (\alpha)\right]$, Eq. (1) becomes

$$
\phi_{F}(t)=\phi_{0}(t)+k_{1} \phi_{1}(t)+k_{2} \phi_{2}(t),
$$

where $\quad \phi_{1}(t)=\sin \phi_{F}(t), \quad \phi_{2}(t)=\cos \phi_{F}(t), \quad k_{1}=$ $C / \sqrt{1+\alpha^{2}}$, and $k_{2}=\alpha C / \sqrt{1+\alpha^{2}}$. Hence, $\alpha$ and $C$ can be determined by

$$
\alpha=\frac{k_{2}}{k_{1}} \quad \text { and } \quad C=\sqrt{k_{1}+k_{2}} .
$$

The phase signal $\phi_{F}(t)$ can be retrieved from an experimental SMI signal $p(t)$ by applying phase unwrapping [19]. Then $\phi_{1}(t)$ and $\phi_{2}(t)$ can be constructed from $\overline{\phi_{F}}(t)$. Our aim is to determine $k_{1}$ and $k_{2}$ from these three signals $\phi_{1}(t), \phi_{2}(t)$, and $\phi_{F}(t)$, which will allow us to work out the values of $\alpha$ and $C$. To this end, let us take the Fourier transform of Eq. (4), yielding the following:

$$
\Phi_{F}(f)=\Phi_{0}(f)+k_{1} \Phi_{1}(f)+k_{2} \Phi_{2}(f),
$$

where $\Phi_{0}(f), \Phi_{1}(f), \Phi_{2}(f)$, and $\Phi_{F}(f)$ are the Fourier transform (complex spectra) of $\phi_{0}(t), \phi_{1}(t), \phi_{2}(t)$, and $\phi_{F}(t)$, respectively. Note that $\Phi_{0}(f), \Phi_{1}(f), \Phi_{2}(f)$, and $\Phi_{F}(f)$ are all complex functions, consisting of a real and an imaginary component. As $\phi_{0}(t)=4 \pi l(t) / \lambda_{0}$, by setting the external target to vibrate in a manner close to simple harmonic vibration, $\phi_{0}(t)$ will be of narrow band in the frequency domain. Due to the nonlinear relationship between $\phi_{0}(t)$ and $\phi_{F}(t)$ shown in Eq. (1), $\Phi_{F}(f)$ [and thus $\Phi_{1}(f)$ and $\Phi_{2}(f)$ ] should spread over a wider frequency range than $\Phi_{0}(f)$ does. Simulations show that, for the cases of the target having simple harmonic vibration or periodic triangular movement, $\Phi_{0}(f)$ should have vanished when the frequency reaches 15 times the fundamental vibration $f_{0}$, while $\Phi_{F}(f)$ still exhibits nonzero components. Hence, we can choose the frequency range $\Omega: f \subset\left(15 f_{0}, f_{M}\right)$, where $f_{M}$ is the highest frequency for $\Phi_{F}(f) \neq 0$. As $\Phi_{0}(f)=0$ but $\Phi_{F}(f) \neq 0$ on $\Omega$, from Eq. (6), we have

$$
\Phi_{F}(f)=k_{1} \Phi_{1}(f)+k_{2} \Phi_{2}(f), \quad \text { where } f \subset \Omega .
$$

Considering Eq. (7) with respect to all the frequencies on $\Omega$, we have the following:

$$
\Psi_{F}=k_{1} \Psi_{1}+k_{2} \Psi_{2},
$$

where $\Psi_{F}=\sum_{f \subset \Omega} \Phi_{F}(f), \Psi_{1}=\sum_{f \subset \Omega} \Phi_{1}(f)$, and $\Psi_{2}=$ $\sum_{f \subset \Omega} \Phi_{2}(f)$.
By separating the real and imaginary parts of Eq. (8) and jointly solving the two equations for $k_{1}$ and $k_{2}$, we have

$$
k_{1}=\frac{\Psi_{F}^{I} \Psi_{2}^{R}-\Psi_{F}^{R} \Psi_{2}^{I}}{\Psi_{1}^{I} \Psi_{2}^{R}-\Psi_{1}^{R} \Psi_{2}^{I}} \quad \text { and } \quad k_{2}=\frac{\Psi_{F}^{R} \Psi_{1}^{I}-\Psi_{R}^{I} \Psi_{1}^{R}}{\Psi_{1}^{I} \Psi_{2}^{R}-\Psi_{1}^{R} \Psi_{2}^{I}}
$$

where superscripts " $R$ " and " $I$ " denote the real and imaginary parts of the relevant complex numbers (e.g., $\Psi_{1}=\Psi_{1}^{R}+j \Psi_{1}^{I}$ ).

With the method presented above, we studied the characteristics of the alpha of a GaAlAs laser diode (HL7851G) with a multiquantum well structure. The lasing wavelength of the laser is around $760 \pm 0.5 \mathrm{~nm}$ when the injection current is within the range from 70 to $140 \mathrm{~mA}$. A loudspeaker was used as the external target, which was driven by a sinusoidal signal of $201 \mathrm{~Hz}$ to provide a periodically varying external optical phase $\phi_{0}(t)$. An attenuator was placed between the laser and the loudspeaker for adjusting the optical feedback strength. During the experiments, the laser temperature was kept at $25 \pm 0.1^{\circ} \mathrm{C}$. SMI signals are detected by a photodetector packaged in the rear of the laser diode.

The first group of experiments was to study the influence of the EOF on the alpha factor. In the experiments, the external cavity was set to be $22 \mathrm{~cm}$ long, and the injection current is set to four fixed points, including 70, 90, 105 , and $140 \mathrm{~mA}$, respectively. At each current point, we adjusted the attenuator in order to change the strength of EOF. When a stable SMI signal was acquired, we employed the proposed method to determine both $C$ and alpha. Following such a procedure, at each of the four injection current points, we obtained 30 pairs of $C$ and alpha with the laser under different EOF levels. The results of the measurement are shown in Fig. 1, which clearly show that the alpha exhibits a wide range of variance (i.e., from 2.5 to 5), and that the alpha value goes up when $C$ increases over the range from 1 to 7 .

In order to investigate the influence of the injection current on the alpha, we conducted another group of experiments. In the experiments, we wish to keep $C$ unchanged and to vary the injection current from 70 to $140 \mathrm{~mA}$ on 15 equally spaced points. However, when changing the current, it is difficult to hold $C$ as a constant. Hence, for each current point, we adjust slightly the attenuator so that the variance of $C$ is kept within a small

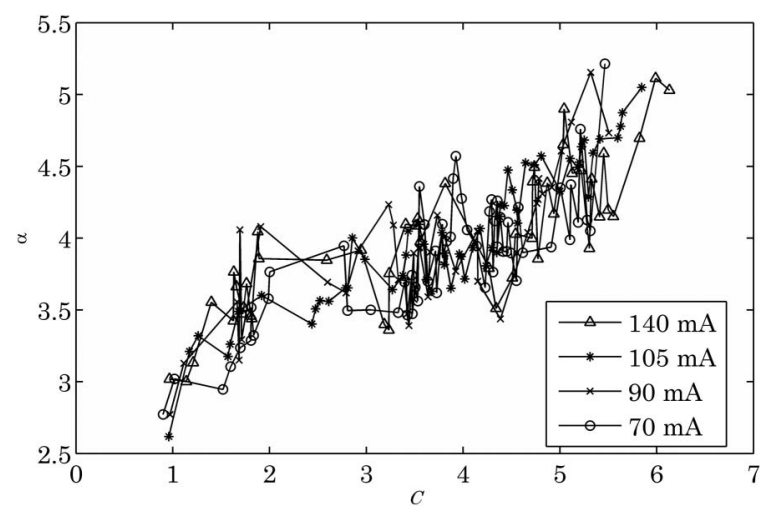

Fig. 1. Influence of feedback parameter $C$ on alpha factor. 


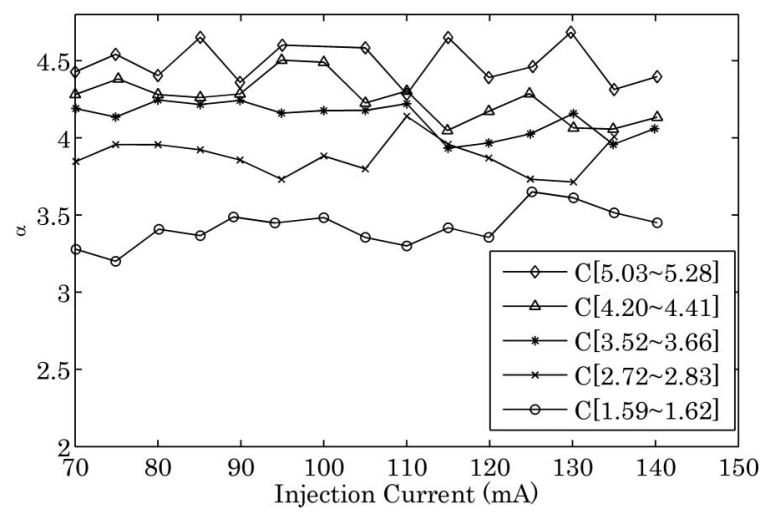

Fig. 2. Relationship between injection current and alpha.

range. Five different ranges of $C$ shown in Fig. 2 were chosen for the investigation on current dependence of the alpha. Within each of the chosen ranges, 15 SMI signals were acquired corresponding to 15 different current points, yielding 15 pairs of alpha and $C$. Figure 2 shows the results of both alpha and $C$ for all the five ranges of $C$, from which we obtained five curves for describing the relationship between alpha and $C$. It can be seen that the five curves all are rather flat, implying that there is not a remarkable dependent relationship between alpha and the inject current. Furthermore, the five curves are laid one on top of another in the order of increasing $C$ value, showing again that the alpha increases with the increase of $C$. Note that the results presented in Figs. 1 and 2 have the same level of accuracy as what is presented in [15] and [18], because they are all based on SMI signals from the same experimental setup.

The contribution in this Letter is twofold. First, it presents a new method for the measurement of the alpha over a wide range of feedback parameter $C$. The proposed method lifts the limitations associated with existing SMI-based methods where a particular approach is only valid for a particular range of $C$. Second, the proposed technique has been employed to investigate the alpha factor of a diode laser with multiple QWs under different optical feedback level. The results revealed a strong correlation between the alpha and $C$; that is, the alpha value goes up with the increase of optical feedback level. It is also discovered that, when keeping $C$ within a small range, the injection current does not have remarkable influence on the alpha factor.

The authors would like to thank Miss Yan Gao for the collection of the experimental data and the numerical computations on the alpha from the data using the proposed method.

\section{References}

1. C. H. Henry, IEEE J. Quantum Electron. 18, 259 (1982).

2. M. Osiniski and J. Buus, IEEE J. Quantum Electron. 23, 9 (1987).

3. T. Fordell and A. M. Lindberg, IEEE J. Quantum Electron. 43, 6 (2007).

4. A. Consoli, B. Bonilla, J. Manuel, G. Tijero, and I. Esquivias, Opt. Express 20, 4979 (2012).

5. G.-J. Provost and F. Grillot, IEEE J. Photon. 3, 476 (2011).

6. Z. Mi and P. Bhattacharya, IEEE J. Quantum Electron. 43, 363 (2007).

7. F. Grillot, N. A. Naderi, M. Pochet, C.-Y. Lin, and L. F. Lester, Appl. Phys. Lett. 93, 191108 (2008).

8. E. Gehriga and O. Hess, Appl. Phys. Lett. 86, 203116 (2005).

9. S. Melnik, G. Huyet, and A. Uskov, Opt. Express 14, 2950 (2006).

10. F. Grillot, B. Dagens, J.-G. Provost, H. Su, and L. F. Lester, IEEE J. Quantum Electron. 44, 946 (2008).

11. K. Kechaou, F. Grillot, J. -G. Provost, B. Thedrez, and D. Erasme, Opt. Express 20, 26062 (2012).

12. R. Lang and K. Kobayashi, IEEE J. Quantum Electron. 16, 347 (1980).

13. G. Acket, D. Lenstra, A. Den Boef, and B. Verbeek, IEEE J. Quantum Electron. 20, 1163 (1984).

14. T. Bosch, in Conference on Optoelectronic and Microelectronic Materials and Devices (IEEE, 2004), pp. 385-392.

15. Y. Yu, G. Giuliani, and S. Donati, IEEE Photon. Technol. Lett. 16, 990 (2004).

16. J. Xi, Y. Yu, J. Chicharo, and T. Bosch, IEEE J. Quantum Electron. 41, 1058 (2005).

17. Y. Yu, J. Xi, J. Chicharo, and T. Bosch, IEEE J. Quantum Electron. 43, 527 (2007).

18. Y. Yu, J. Xi, and J. Chicharo, Opt. Express 19, 9582 (2011).

19. Y. Fan, Y. Yu, J. Xi, and J. Chicharo, Appl. Opt. 50, 5064 (2011). 